Jantien Visser, M.D.

Danielle Cohen, M.D.

Kitty W.M. Bloemenkamp, M.D., Ph.D.

Leiden University Medical Center

Leiden, the Netherlands

k.w.m.bloemenkamp@lumc.nl

No potential conflict of interest relevant to this letter was reported.

THE AUTHORS REPLY: We agree that both the Anticoagulants for Living Fetuses (ALIFE) study (Current Controlled Trials number, ISRCTN58496168) and the Scottish Pregnancy Intervention Study (ISRCTNO6774126) ${ }^{1}$ have investigated a combination of low-molecular-weight heparin and aspirin, and that a detrimental effect of aspirin cannot be entirely ruled out. Therefore, studies of the effect of low-molecular-weight heparin alone in women with recurrent miscarriage would be interesting. We disagree that a double-blind design is always necessary. Rather, concealment of study-drug assignment is the quality item in randomized, controlled trials that may lead to the most severely biased effect estimates if it is inadequate. ${ }^{2}$ In the ALIFE study, all randomly assigned women were offered similar standards of care, thus avoiding performance bias. The standard of care in the ALIFE study involved a low threshold for visits and ultrasonography in early pregnancy; these can be considered a form of psychological support.

Currently, no consensus exists on the definition of recurrent miscarriage. Guidelines published on this topic and their respective definitions vary with regard to the number of preceding miscarriages as well as the sequence of previous pregnancies. The American College of Obstetricians and Gynecologists defines recurrent miscarriage as two or more consecutive miscarriages, the European Society of Human Reproduction and Embryology defines it as three or more consecutive miscarriages, and the Royal College of Obstetri- cians and Gynaecologists defines it as three or more miscarriages. This discrepancy is due to a lack of evidence with regard to the role of risk factors such as the number of preceding miscarriages. Maternal age has not been described in any of the above-mentioned guidelines. The discrepancy in definition has led to an ongoing discussion regarding which couples should receive the diagnosis of recurrent miscarriage and when to start the diagnostic workup.

The potential dilutive effects of recruiting women with two miscarriages and advanced maternal age were fully taken into account when our sample-size calculation was performed. This consideration was mainly based on the study by Brigham et al., ${ }^{3}$ a large cohort study based on the same patient group, including women with two miscarriages and advanced maternal age.

We agree with Visser et al. that a meta-analysis of data from individual patients could provide important information regarding a differential effect of therapy in women with a history of two rather than three miscarriages. In the ALIFE trial, the exclusion of women with "only" two preceding miscarriages or women 36 years of age or older would have resulted in a missed opportunity.

Stef P. Kaandorp, M.D.

Mariëtte Goddijn, M.D., Ph.D.

Saskia Middeldorp, M.D., Ph.D.

Academic Medical Center

Amsterdam, the Netherlands

m.goddijn@amc.uva.nl

Since publication of their article, the authors report no further potential conflict of interest.

1. Clark P, Walker ID, Langhorne P, et al. SPIN (Scottish Pregnancy Intervention) study: a multicenter, randomised controlled trial of low-molecular-weight heparin and low-dose aspirin in women with recurrent miscarriage. Blood 2010;115:4162-7.

2. Jüni $P$, Altman DG, Egger M. Systematic reviews in health care: assessing the quality of controlled clinical trials. BMJ 2001; 323:42-6.

3. Brigham SA, Conlon C, Farquharson RG. A longitudinal study of pregnancy outcome following idiopathic recurrent miscarriage. Hum Reprod 1999;14:2868-71.

\title{
Cost Consciousness and Medical Education
}

TO THE EDITOR: We commend Cooke's efforts in her Perspective article (April 8 issue) ${ }^{1}$ to increase readers' awareness of the near-universal ignorance of actual costs associated with the delivery of medical care..$^{2-4}$ This lack of cost awareness affects all other components of the price equation, rendering us incapable of understanding the true economic value of medical care. It also makes us unable to engage efficiently in any potential negotiation. ${ }^{5}$ As end consumers, we as physicians must educate ourselves to know better prices will never drop (even if the true cost is low) because of our lack of cost consciousness. The asymmetry between physicians and adminis- 
trators in knowledge about costs mirrors the asymmetry between patients and physicians regarding information about medical care.

We must change the way we educate newer generations of health care providers, and educators themselves must be prepared to teach new guidelines. Innovation is needed, not just in terms of advanced technologies, research, and newer therapies, but also in the way we teach newer generations about proper business management and instill administrative survival skills. Medical schools and residency programs should leverage their curriculum with partner business schools to address these issues.

Homero Rivas, M.D., M.B.A.

John M. Morton, M.D., M.P.H.

Thomas M. Krummel, M.D.

Stanford University School of Medicine

Stanford, CA

hrivas@stanford.edu

No potential conflict of interest relevant to this letter was reported.

1. Cooke M. Cost consciousness in patient care - what is medical education's responsibility? N Engl J Med 2010;362:1253-5.

2. Schilling UM. Cutting costs: the impact of price lists on the cost development at the emergency department. Eur J Emerg Med 2010 January 20 (Epub ahead of print).

3. Idem. Cost awareness among Swedish physicians working at the emergency department. Eur J Emerg Med 2009;16:131-4.

4. Allan GM, Lexchin J, Wiebe N. Physician awareness of drug cost: a systematic review. PLoS Med 2007;4(9):e283.

5. Eisenhauer JM, Principe KE. Price knowledge and elasticity. J Empir General Market Sci 2009;12:1-20.

TO THE EDITOR: In addition to the three factors noted by Cooke, I wish to add that the extravagant use of health care is being driven in many instances by the psychological comfort of physicians. In teaching hospitals, blood transfusions and costly biologic agents are frequently administered so that the doctor will feel better. Examples include the use of blood and clotting factors before bedside procedures in patients with trivial abnormalities of coagulation, transfusions to "treat worrisome laboratory values" in the absence of clinical need, and the expanding use of extremely expensive biologic agents that are prescribed offlabel to reassure physicians. Such motivations are distinct from patient advocacy. When these expenditures are multiplied by all specialties, the total is beyond staggering. Dealing with medical uncertainty is not easy, but the psychological comfort that physicians gain by pursuing every imaginable diagnosis or treatment is no justification for ruinous expenditures on health care. When placed in the greater context of health care available for most of the world's population, the extravagance is far beyond unjustifiable.

Walter Dzik, M.D.

Massachusetts General Hospital

Boston, MA

sdzik@partners.org

No potential conflict of interest relevant to this letter was reported.

TO THE EDITOR: Cooke rightly points at the moral, societal, and economic reasons for educating students in medically sound strategies for cost containment, and we wholeheartedly support her argument for a value-based approach to choosing treatment. However, we do not agree that patientcentered care contributes to increasing health care costs. In fact, patient-centered care might hold the solution.

We physicians conceive of ourselves as advocates for each patient, whereas research shows that health care professionals agree little with patients and family caregivers on treatment goals. ${ }^{1,2}$ It is therefore a misconception that doctors know best what creates value for a patient. Patient-centered care, shared decision making, and collaborative goal setting can help us understand what a patient values most. Will this result in additional health care spending? We doubt it. Although strong evidence on the effectiveness of collaborative goal setting is lacking, ${ }^{3}$ its promise for improving cost-effectiveness is evident: it would do so first by increasing patient satisfaction and adherence ${ }^{2,4}$ and second by ensuring that the limited available resources are allocated to the care that matters most to the patient.

Sarah H.M. Robben, M.D.

René J.F. Melis, M.D., Ph.D.

Marcel G.M. Olde Rikkert, M.D., Ph.D.

Radboud University Nijmegen Medical Center

Nijmegen, the Netherlands

s.robben@ger.umcn.nl

No potential conflict of interest relevant to this letter was reported.

1. Glazier SR, Schuman J, Keltz E, Vally A, Glazier RH. Taking the next steps in goal ascertainment: a prospective study of patient, team, and family perspectives using a comprehensive standardized menu in a geriatric assessment and treatment unit. J Am Geriatr Soc 2004;52:284-9.

2. Bogardus ST, Bradley EH, Williams CS, Maciejewski PK, van Doorn C, Inouye SK. Goals for the care of frail older adults: do caregivers and clinicians agree? Am J Med 2001;110:97-102.

3. Bodenheimer T, Handley MA. Goal-setting for behavior change in primary care: an exploration and status report. Patient Educ Couns 2009;76:174-80.

4. Bogardus ST Jr, Bradley EH, Williams CS, Maciejewski PK, 
Gallo WT, Inouye SK. Achieving goals in geriatric assessment: role of caregiver agreement and adherence to recommendations. J Am Geriatr Soc 2004;52:99-105.

TO THE EDITOR: Cooke believes that medical educators have a moral obligation to address cost, and I would argue that this should not be an issue whose discussion is left to the discretion of medical educators. Education about cost should be standardized, integrated, and clearly outlined in the medical curriculum. Linking evidence-based medicine with economic evaluation and communication (negotiation) skills should be clearly highlighted in the curriculum. Recent research articles $^{1,2}$ have addressed this concept and could be used as resources for students' discussion.

In addition, Cooke did not highlight the importance of patient education in reducing costs or the value of rehabilitation and preventive programs in minimizing the need for hospital care ${ }^{3}$ - an important consideration for patients with diabetes, hypertension, chronic respiratory problems, or obesity, for example. ${ }^{4}$ Medical education should place more emphasis on patient education and preventive programs.

Samy A. Azer, M.D., Ph.D.

King Saud University College of Medicine

Riyadh, Saudi Arabia

azer2000@optusnet.com.au

No potential conflict of interest relevant to this letter was reported.

1. Miller LA, Wade R, Dai D, Cziraky MJ, Romaswamy K, Panjabi S. Economic evaluation of four angiotensin II receptor blockers in the treatment of hypertension. Curr Med Res Opin 2010;26:1307-20.

2. Winchester DE, Wymer DC, Shifrin RY, Kraft SM, Hill JA. Responsible use of computed tomography in the evaluation of coronary artery disease and chest pain. Mayo Clin Proc 2010; 85:358-64.

3. Rasekaba TM, Williams E, Hsu-Hage B. Can a chronic disease management pulmonary rehabilitation program for COPD reduce acute rural hospital utilization? Chron Respir Dis 2009;6: 157-63.

4. 4. Trueman P, Haynes SM, Lyons GF, et al. Long-term cost effectiveness of weight management in primary care. Int J Clin Pract 2010;64:775-83.

THE AUTHOR REPLIES: The correspondents all add important points to my brief essay in support of the contention that medical education should address cost. Rivas et al. note that it can be difficult to obtain information on charges. Furthermore, the weak relationship among what is charged, what is paid, and what a test or treatment actually costs hinders the development of cost consciousness. ${ }^{1}$ Robben et al. challenge what they took to be my assertion that patient-centeredness increases the cost of care; I appreciate the opportunity to clarify. Like these correspondents, I find evidence that patient-centeredness may decrease the cost of care. ${ }^{2}$ However, if a "customer satisfaction" orientation is confused with patientcenteredness and is compounded by lack of time for fully eliciting patients' values and exploring risks and benefits, busy physicians may simply accede to requests for minimally beneficial care. ${ }^{3}$ This is entirely different from shared decision making and collaborative goal setting.

Dzik calls our attention to the physician as the driver in wasteful care. In addition to ordering tests as a way of reducing psychological discomfort associated with uncertainty, many physicians would implicate the tort system as a cause of wasteful "defensive medicine." ${ }^{4}$ Azer correctly notes that innovative delivery systems that support patients' self-management and matching patients' needs with appropriate caregivers can prevent the development of disease and provide care in settings outside the acute care hospital at lower cost. ${ }^{5}$

As we move to reform health care in the United States, there are substantial opportunities to build into medical education the knowledge and skills that are required for the provision of cost-conscious care. Imagine, for example, a computerized order-entry system that displays the charge for each drug or test at the time that it is ordered and that informs the physician of the daily charges for each patient and the cost of the hospitalization, as compared with the average cost for the principal diagnosis-related group. However, other issues that these letters raise cannot be easily addressed in the inpatient setting. Learners following patients over time in a well-functioning delivery system, whether it be an accountable care organization or a patient-centered medical home in a "good medical neighborhood," will learn skills in shared decision making, participate in collegial teams of health care professionals, and come to appreciate the benefits of judiciously designed and collaboratively implemented diagnostic and therapeutic strategies for patients and the health care system.

\section{Molly Cooke, M.D.}

University of California, San Francisco

San Francisco, CA 
Since publication of her article, the author reports no further potential conflict of interest.

1. Robinson JC. Price transparency begins at home. Front Health Serv Manage 2007;23:25-8.

2. O'Connor AM, Bennett CL, Stacey D, et al. Decision aids for people facing health treatment or screening decisions. Cochrane Database Syst Rev 2009;3:CD001431.

3. Gellad ZF, Lyles KW. Direct-to-consumer advertising of pharmaceuticals. Am J Med 2007;120:475-80.
4. Hermer LD, Brody H. Defensive medicine, cost containment, and reform. J Gen Intern Med 2010;25:470-3.

5. Grumbach K, Bodenheimer T, Grundy P. The outcomes of implementing patient-centered medical home interventions: a review of the evidence on quality, access and costs from recent prospective evaluation trials, August 2009. Washington, DC: Patient-Centered Primary Care Collaborative, 2009. (Accessed August 5, 2010, at http://www.pcpcc.net/files/pcmh_evidence_ outcomes_2009.pdf.)

\section{Reference Range for Cerebrospinal Fluid Opening Pressure in Children}

TO THE EDITOR: A reference range for cerebrospinal fluid (CSF) opening pressure in children undergoing diagnostic lumbar puncture has not been established. ${ }^{1}$ The influence of age, body-mass index (BMI), and depth of sedation on opening pressure in children is also uncertain. ${ }^{2}$

We conducted a 2-year, single-center prospective study of CSF opening pressure in children undergoing lumbar puncture as part of their routine clinical care. Our objective was to establish a useful, clinically relevant reference range for opening pressure and a threshold value for abnormally elevated opening pressure (in the 90th percentile or higher, defined a priori). Our reference group consisted of children between 1 and 18 years of age, none of whom was taking a medication or had signs of a disease or condition that would alter opening pressure on lumbar puncture (e.g., papilledema, hydrocephalus, cerebral edema, Chiari malformation, meningitis, or use of diuretics). The procedure was performed with subjects in the lateral recumbent position, and the opening pressure was measured with the use of a standard manometer. (For enrollment criteria, methods, and results, see the Supplementary Appendix, available with the full text of this letter at NEJM .org.) Reference-range parameters for opening pressure were determined with the use of standard descriptive statistics, whereas associations between age, BMI, and depth of sedation were determined using linear regression analysis.

We enrolled 472 subjects during the study period, and 197 (41.7\%) met the inclusion criteria for the reference-range population. The threshold for an abnormally elevated opening pressure, deter- mined on the basis of the 90th percentile for all patients in the reference population, was $28 \mathrm{~cm}$ of water (Fig. 1A). The threshold for an abnormally reduced pressure in the 10th percentile was $11.5 \mathrm{~cm}$ of water. Subjects placed under moderate to deep sedation during lumbar puncture had a slightly higher opening pressure as compared with those not receiving any sedative medication $(\beta=3.459, \mathrm{P}=0.002)$ (Fig. 1B). Similar to studies in adults, ${ }^{3,4}$ our study showed a small yet statistically significant positive relationship between opening pressure and BMI $(\beta=0.313, \mathrm{P}=0.002)$ (Fig. 1C). Subject age was not associated with increased opening pressure ( $\mathrm{P}=0.43)$ (Fig. 1D). Given the significant association between depth of sedation and BMI on opening pressure, a post-hoc analysis of opening pressure percentiles was calculated for the 52 subjects who received minimal or no sedation and were not classified as obese, resulting in a 90th percentile of $25 \mathrm{~cm}$ of water.

This study systematically defined the reference range for CSF opening pressure and the threshold value for abnormally elevated opening pressure in a representative pediatric population (in whom evident pathology was excluded) undergoing diagnostic lumbar puncture. It is our opinion that previous estimates of what constitutes an "abnormal" opening pressure, such as $20 \mathrm{~cm}$ of water, ${ }^{1,5}$ should be reconsidered, and that for most children an opening pressure above $28 \mathrm{~cm}$ of water should be considered elevated.

Robert A. Avery, D.O.

Samir S. Shah, M.D., M.S.C.E.

Daniel J. Licht, M.D.

Jeffrey A. Seiden, M.D. 